Trans

continentales
Transcontinentales

Sociétés, idéologies, système mondial

$8 / 9 \mid 2010$

Des migrations aux circulations transnationales

\title{
L'émigration indonésienne en Malaysie
}

Enjeux religieux, culturels et politiques contemporains

Indonesian emigration in Malaysia. Contemporary religious, cultural and political issues

\section{Rémy Madinier}

\section{OpenEdition}

\section{Journals}

Édition électronique

URL : http://journals.openedition.org/transcontinentales/792

DOI : $10.4000 /$ transcontinentales.792

ISBN : 978-2-7351-1557-0

ISSN : 1775-397X

Éditeur

Editions de la maison des sciences de l'homme

Édition imprimée

Date de publication : 31 décembre 2010

ISSN : 1950-1684

Référence électronique

Rémy Madinier, «L'émigration indonésienne en Malaysie », Transcontinentales [En ligne], 8/9 | 2010, document 7, mis en ligne le 31 décembre 2010, consulté le 08 septembre 2020. URL : http:// journals.openedition.org/transcontinentales/792 ; DOI : https://doi.org/10.4000/transcontinentales. 792

Ce document a été généré automatiquement le 8 septembre 2020

Tous droits réservés 


\section{L'émigration indonésienne en Malaysie}

Enjeux religieux, culturels et politiques contemporains

Indonesian emigration in Malaysia. Contemporary religious, cultural and political issues

Rémy Madinier

1 L'histoire récente des migrations indonésiennes vers la Malaisie voisine est particulière parce qu'elle met en présence deux États-nations appartenant à une même aire culturelle et aux destins longtemps liés. Séparés par la colonisation puis par des développements économiques divergents, les deux pays déclinent désormais leurs relations sur un mode inégalitaire. L'analyse de ces mouvements migratoires dans une perspective historique permet d'en comprendre les surprenants prolongements culturels, politiques et même religieux, et d'éclairer le rôle de ces phénomènes dans la crise actuelle des relations entre ces deux voisins (carte 1). 


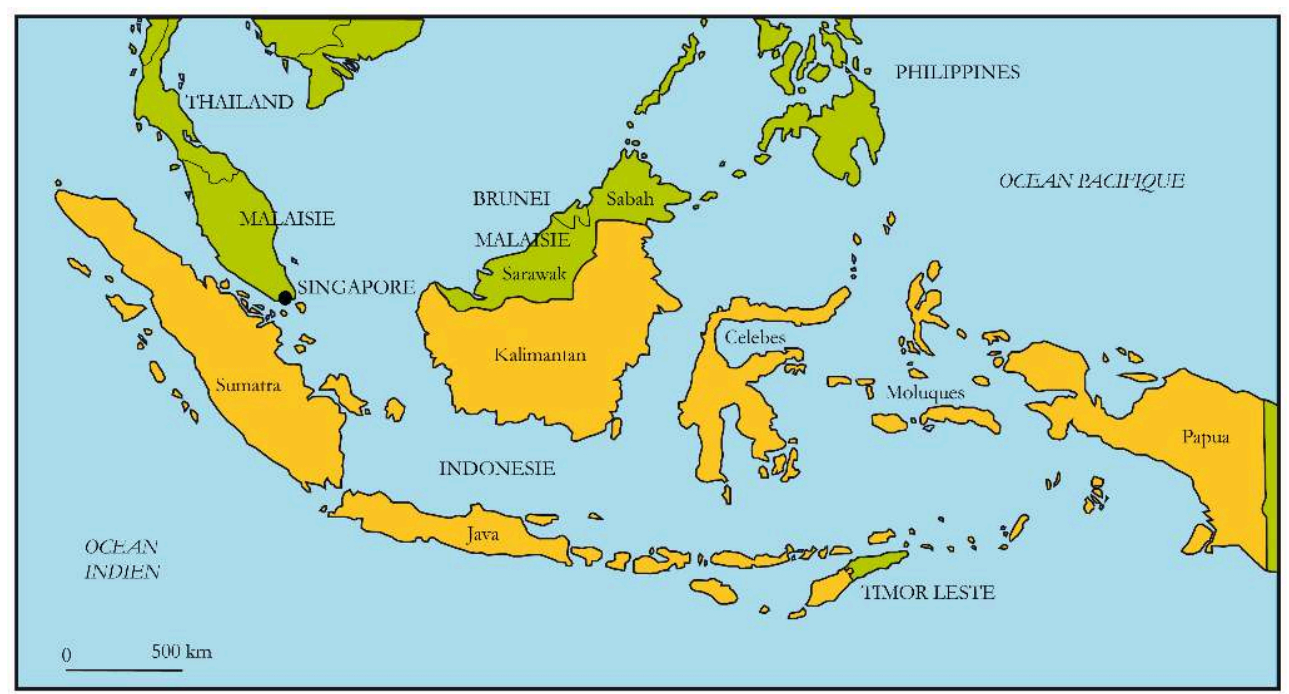

L'Indonésie et ses voisins

\section{Des migrations traditionnelles au sein d'une même aire de civilisation}

2 Les migrations de populations entre les territoires appartenant aujourd'hui à l'Indonésie et à la Malaisie s'inscrivent dans une histoire de temps longs. Ces deux États-nations appartiennent en effet à une aire de civilisation, le monde malais, définie par une communauté culturelle, religieuse et linguistique renforcée par des structures politiques communes ${ }^{1}$.

3 Tant à la période hindo-bouddhiste qu'après l'arrivée de l'islam, des royaumes ont gouverné des territoires situés sur les deux rives du détroit de Malacca ${ }^{2}$. Entre le viII ${ }^{\mathrm{e}}$ et le $\mathrm{XII}^{\mathrm{e}}$ siècle, par exemple, la thalassocratie bouddhiste de Sriviyaya, dont la capitale était située dans la région de Palembang, à Sumatra, étendit son influence sur une grande partie de l'Insulinde et de la péninsule malaise. Et, lorsque le modèle du sultanat se répandit, à partir de la fin du XIII ${ }^{\mathrm{e}}$ siècle, plusieurs d'entre eux (Aceh à Sumatra, Johore Baru en péninsule) ont gouverné des territoires de part et d'autre du détroit de Malacca (carte 2). 


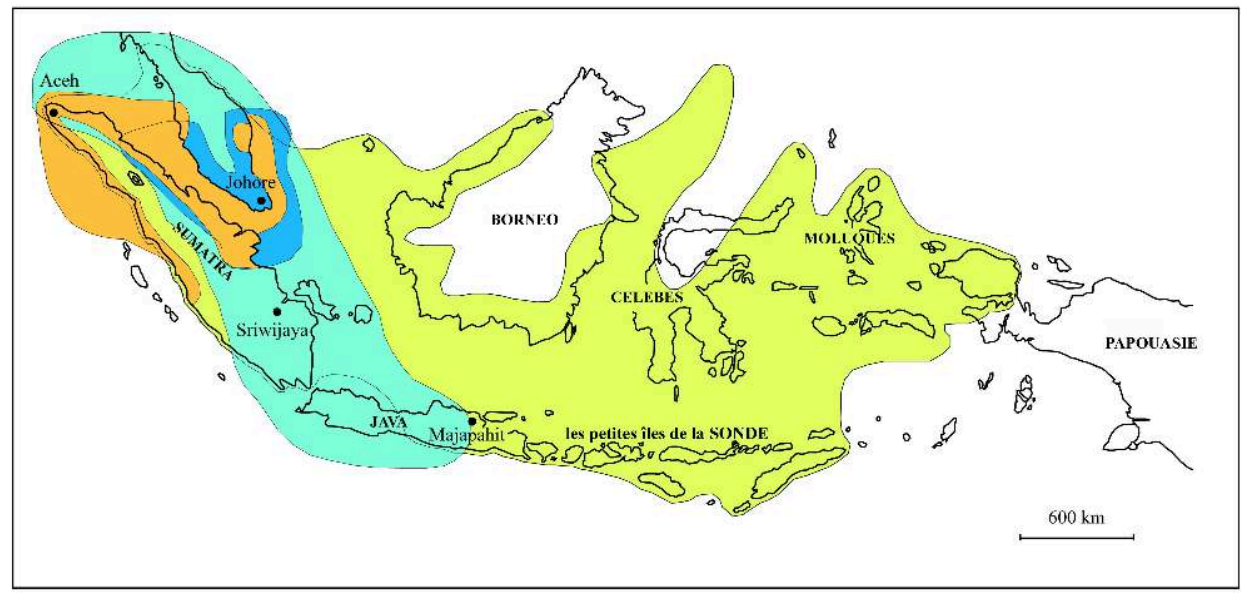

ire d'influence de Sriwijaya au 12ème Siècle

Aire d'influence de Majapahit au 14 ème Siècle

Aire d'influence de .Johore au ló̀me Siècle

Aire d'influence d'Aceh au 17 ème Siècle

Les aires d'influence des principaux empires du monde insulindien

Conception Remy Madinier (CNRS-IRASEC), réalisation Ade Pristic Wahyo (EFEO)

4 Traditionnellement, dans cet espace qui fut longtemps couvert par d'épaisses forêts infranchissables, la mer reliait bien plus qu'elle ne séparait ${ }^{3}$. Outre le modèle politique du sultanat, l'islam contribua à diffuser, dans l'ensemble de l'Archipel, le malais qui devint plus tard la langue officielle des deux État voisins. Les réseaux commerciaux qui se formèrent alors favorisèrent les migrations. Au sein de principautés où la maind'œuvre était rare, la capacité des souverains à attirer, volontairement ou par la contrainte, les forces nécessaires aux difficiles travaux de défrichement, témoignait de leur puissance. La nature souple et mouvante - sous l'effet des changements d'alliance entre royaumes - des frontières encouragea échanges commerciaux et déplacements de population, contribuant ainsi à l'unité culturelle d'une région par ailleurs très ouverte sur le monde ${ }^{4}$. Bien avant la colonisation, des circuits migratoires se mirent en place. Ils reliaient plus particulièrement Sumatra à la péninsule malaise toute proche, mais des populations plus lointaines (venant de Java ou des Célèbes) les empruntèrent également (carte 3). 


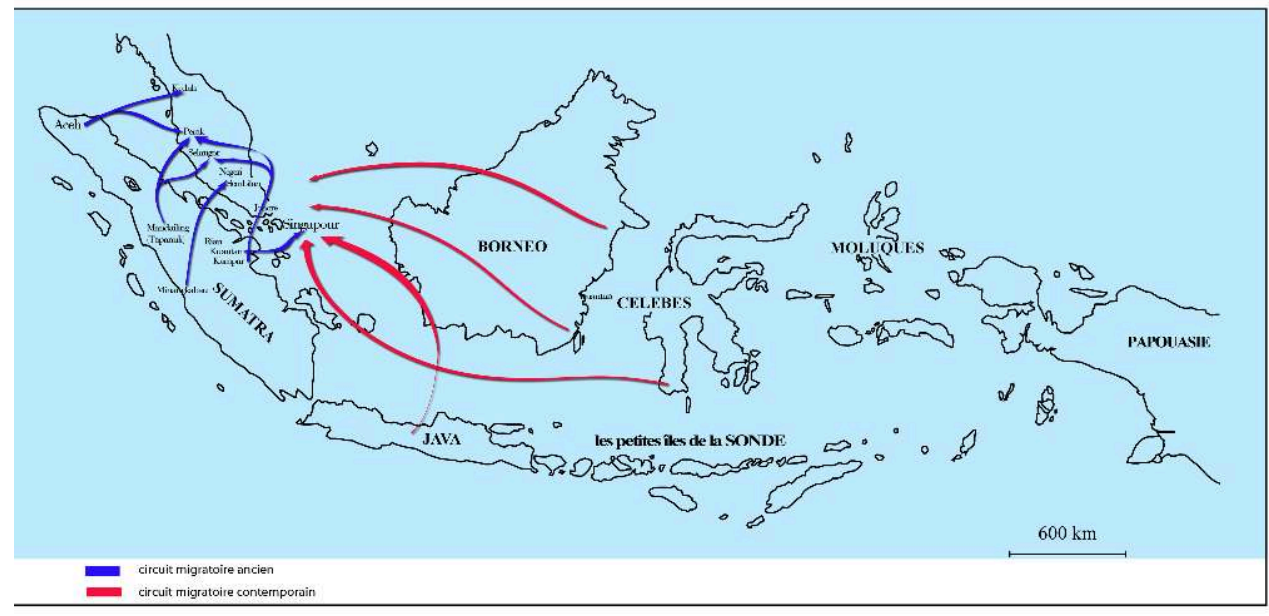

Migrations anciennes et contemporaines entre Indonésie et Malaisie

Conception Remy Madinier (cNRS-IRASEC), réalisation Ade Pristic Wahyo (EFEO)

Durant cette période, la question de l'identité ne se posait bien sûr pas en termes de frontières : la «malayité » métisse et plurielle par nature (melayu kacukan) se définissait à la fois par sa capacité à intégrer des éléments extérieurs et par la possibilité pour toutes les ethnies de se voir reconnues comme malaises ${ }^{5}$.

Le moment colonial de l'Asie du Sud-Est nousantarienne ne bouleversa pas les itinéraires de migration, bien au contraire. D'abord parce que cette colonisation fut relativement tardive : bien qu'installés à Batavia, la future Jakarta, dès le début du XviI ${ }^{\mathrm{e}}$ siècle, les Hollandais n'achevèrent de contrôler l'espace indonésien que dans la première décennie du $\mathrm{xx}^{\mathrm{e}}$ siècle. Aceh, en particulier, ne fut soumis qu'au terme d'une longue et difficile guerre (1873-1904). Les Britanniques, quant à eux, ne prirent pied dans la région qu'à la fin du XVIII ${ }^{\mathrm{e}}$ siècle, en acquérant, entre 1786 et 1824, plusieurs établissements (Penang, Malacca, Singapour) qui leur permettaient de contrôler la principale route maritime de la région, celle du détroit séparant Sumatra de la péninsule. Le traité anglo-hollandais de 1824 démarqua les sphères d'influence respectives des deux puissances. Il traça une frontière au milieu du détroit de Malacca, mais ne mit pas fin pour autant aux déplacements de populations. Afin de mettre en valeur les territoires peu peuplés de la péninsule malaise (300 000 habitants en 1835), sur lesquels leur influence s'étendit progressivement, les anglais organisèrent des migrations massives : Chinois, Indiens (d'ethnie tamoule à $85 \%$ ), mais aussi populations de Java et de Sumatra furent encouragés à venir travailler dans les plantations d'hévéas et les mines d'étain. Au XIX ${ }^{e}$ siècle, il demeurait encore très facile pour les migrants originaires d'Insulinde de s'installer définitivement dans la péninsule: pour s'approprier une terre vacante, il suffisait de la défricher et de le signaler à un représentant $\mathrm{du}$ gouvernement colonial. Les Britanniques encouragèrent même ces installations en prenant à leur charge les travaux nécessaires à la mise en culture des zones marécageuses ${ }^{6}$. Plusieurs ethnies originaires de Sumatra continuèrent de jouer un rôle privilégié dans ces migrations: Les Kampar partaient exploiter de grandes plantations dans les États malais de Perak et de Selangor, les Minang se dirigeaient, quant à eux, essentiellement vers Negeri Sembilan où ils parvinrent, en s'éloignant de 
la côte, à retrouver des conditions favorables à la riziculture. Ces migrants sumatranais ne considéraient pas la péninsule malaise comme une terre étrangère mais comme une région où certains de leurs ancêtres s'étaient installés et dont ils se sentaient souvent plus proches que des autres régions des Indes néerlandaises ${ }^{7}$.

$7 \mathrm{Au}$ cours du XIX ${ }^{\mathrm{e}}$ siècle, la part des Javanais dans ces migrations se fit de plus en plus importante : le dynamisme démographique de l'île (3 millions d'habitants au début du $\mathrm{XIX}^{\mathrm{e}}$ siècle, 41 millions en 1930) obligea en effet le colonisateur hollandais à organiser des migrations intérieures et à tolérer l'émigration vers la Malaisie. Les bureaux du gouverneur général des Indes néerlandaises à Penang ou à Singapour furent ainsi chargés de fournir des permis aux planteurs malais souhaitant embaucher des Javanais ${ }^{8}$. Des réseaux migratoires professionnels et non plus familiaux apparurent: ainsi, les intermédiaires qui, dans les deux dernières décennies du $\mathrm{xIX}^{\mathrm{e}}$ siècle, envoyèrent massivement des Javanais effectuer le pèlerinage à la Mecque, furent également ceux qui organisèrent ensuite leur embauche dans les plantations de la péninsule.

8 Après la première guerre mondiale, alors que la future autonomie des colonies commençait à être discutée, ces migrations prirent une dimension politique. Les Britanniques qui, on l'a vu, avaient profondément modifié l'équilibre ethnique des États de la péninsule malaise en encourageant les arrivées massives d'Indiens et de Chinois, souhaitèrent néanmoins assurer une certaine prééminence, en particulier en termes de droits fonciers, aux Malais et aux populations qu'ils assimilaient à des Malais. Ils contribuèrent ainsi à définir la "malayité » comme une identité désormais plus précise et plus fermée, investie d'une légitimité supérieure à celle des autres "races» identifiées dans leurs recensements9.

9 De ce fait, à partir des années 1930, ils favorisèrent, par une politique de quotas, les migrants venant des Indes néerlandaises. Ces derniers, même s'ils ne bénéficiaient pas immédiatement des droits confiés aux citoyens malaisiens, étaient considérés comme des Malais, par opposition aux Chinois et aux Indiens ${ }^{10}$.

\section{Les indépendances et la rigidification des frontières}

10 Ces distinctions ethnico-culturelles prirent une grande importance au lendemain de la seconde guerre mondiale : comme le montra le recensement de 1947 dans la péninsule, l'impératif démocratique était désormais en contradiction avec le souhait des Britanniques d'assurer le contrôle du pouvoir politique aux Malais (le contrôle de l'économie revenant, de fait aux autres ethnies).

11 Sur un total de six millions d'habitants, dont un à Singapour, la population de la péninsule était répartie de la manière suivante :

\begin{tabular}{|c|c|c|c|}
\hline & Singapour & Malaisie sans Singapour & Total péninsule \\
\hline \hline Chinois & $78 \%$ & $38 \%$ & $44,7 \%$ \\
\hline \hline Malais & $12 \%$ & $49,5 \%$ & $43,5 \%$ \\
\hline \hline Indiens & $7 \%$ & $11 \%$ & $10,3 \%$ \\
\hline
\end{tabular}



réunissant Singapour et les autres territoires de la péninsule malaise sans remettre en cause le dogme de la suprématie politique malaise. Par conséquent, en 1957, ils accordèrent l'indépendance aux États de la péninsule mais conservèrent Singapour dans leur giron. En 1963, cependant, l'émancipation de la cité-État étant devenue inévitable, un élargissement de la fédération l'intégra, en même temps que les anciens territoires britanniques de Bornéo, dans un nouvel ensemble. Afin de préserver la domination politique des Malais sur les Chinois et les Indiens, on accorda aux habitants $\mathrm{du}$ Sabah et du Sarawak (considérés comme Malais dans leur très grande majorité) une représentation au parlement bien supérieure, en proportion, à celle des Chinois de Singapour ${ }^{11}$. Ces graves entorses au principe démocratique, instituées au nom d'un communautarisme culturel et religieux inspiré de l'ère coloniale, hypothéquèrent le bon fonctionnement du nouvel État: en 1965, Singapour fut expulsé de la fédération de Malaisie qui prit alors ses contours définitifs.

13 En plus des problèmes internes, ce processus de construction nationale opposa la Malaisie naissante à son voisin indonésien. Au sein des Indes néerlandaises, une partie du courant nationaliste s'était en effet construit autour de l'idée d'une grande Indonésie sublimant les frontières coloniales ${ }^{12}$. Ce thème ressurgit à la fin des années 1950, du fait de la nouvelle indépendance malaisienne mais aussi à cause de la surenchère nationaliste du régime de Soekarno qui cherchait alors un exutoire à sa dérive autoritaire. Entre 1963 et 1965, les deux pays connurent une période dite de confrontation (Konfrontasi) durant laquelle bruits de bottes et manœuvres d'intimidations créèrent un climat défavorable à la poursuite des migrations.

\section{Le renouveau migratoire sur un mode inégalitaire}

14 Une fois les tensions apaisées, deux éléments contribuèrent à la reprise de migrations massives vers la péninsule malaise, toujours favorisées par le différentiel démographique entre les deux pays.

15 En premier lieu, la défense de l'identité malaise demeurait un élément central de la politique malaisienne. Dans cette perspective, les responsables malais se montrèrent accueillants à l'égard de populations susceptibles de renforcer leur domination. Les dirigeants des partis chinois et indiens accusèrent d'ailleurs souvent les politiciens de l' United Malay National Organisation (UMNo), le parti au pouvoir, de favoriser une immigration indonésienne (légale ou non) afin de faire évoluer le rapport de force ethnique en leur faveur ${ }^{13}$.

16 En second lieu, les évolutions économiques et démographiques des deux voisins encouragèrent la reprise des migrations. Prolongeant le modèle britannique d'une économie fondée sur l'exploitation d'une main-d'œuvre bon marché dans des plantations tournées vers l'exportation ${ }^{14}$, la Malaisie parvint à s'assurer une croissance beaucoup plus rapide que celle de son voisin indonésien. À partir des années 1970, son insertion réussie dans la nouvelle géographie industrielle de l'Asie du Sud-Est fit d'elle l'une des zones de délocalisation privilégiée des pays industrialisés. Fondée sur les différentiels de développement et donc de coût de la main-d'oeuvre, une division internationale $\mathrm{du}$ travail s'opéra à l'échelle de la région. Le gouvernement singapourien, cherchant à limiter l'immigration de travailleurs peu qualifiés sur son

Transcontinentales, $8 / 9$ | 2010 
territoire, encouragea l'installation de ses unités industrielles dans les régions voisines de la Malaisie. Celles-ci durent alors faire appel à l'émigration indonésienne pour occuper les emplois les plus ingrats dont les Malaisiens ne voulaient plus ${ }^{15}$.

17 L'Indonésie, de son côté, faute de pouvoir accueillir ces industries sur son sol, s'organisa pour exporter sa main-d'œuvre: elle créa le statut de travailleur international (Tukang Kerja International, ТкI) et inscrivit dans ses plans quinquennaux des objectifs croissants d'envois de travailleurs à l'étranger (100 000 personnes pour le plan de 1979-1984; 2,8 millions pour celui de 1998-2003) ${ }^{16}$. La Malaisie prit une place de plus en plus importante dans ces migrations et, depuis 2004, elle est devenue la première destination pour les Indonésiens avec 1,3 millions d'émigrants légaux et environ 700000 d'illégaux ${ }^{17}$. Cette immigration est également importante puisque $65 \%$ des migrants étrangers présents sur son sol sont de nationalité indonésienne ${ }^{18}$.

Mais cette émigration indonésienne en Malaisie avait désormais changé de nature : très longtemps symbole d'une libre circulation des hommes au sein d'une aire de civilisation commune, elle devint, avant tout, la conséquence d'un différentiel de développement entre deux pays, comparables quelques décennies plus tôt. Réduite à une fonction économique, bâtie sur un mode inégalitaire, la migration fut dorénavant plus douloureuse pour les Indonésiens. Leur installation définitive en Malaisie par l'acquisition de terres au nom d'une lignée ancestrale devint quasiment impossible.

Plus jeunes, les migrants cherchent seulement, en effectuant quelques années durant des travaux ingrats, à accumuler le pécule nécessaire à leur établissement dans leur région d'origine. Mal perçus par les Malaysiens qui les accusent souvent des divers maux que l'on prête volontiers aux immigrés de fraîche date (prostitution, mauvaise moralité, vol, faible conscience religieuse, etc.), ces travailleurs indonésiens se retrouvent donc fréquemment en situation de faiblesse ${ }^{19}$, d'autant qu'il s'agit de plus en plus de femmes et de clandestins. La féminisation de la main-d'œuvre s'est en effet développée avec les nouveaux types d'emplois proposés aux migrants: aux travaux agricoles dans les plantations ont succédé ceux de nouveaux secteurs industriels (textile, électronique, etc.) et, surtout, des emplois de service à la personne où les Indonésiennes représentent $90 \%$ des effectifs ${ }^{20}$. Situés dans la sphère privée, ces emplois ne bénéficièrent longtemps d'aucune protection légale.

20 La rigidification progressive des frontières contribua par ailleurs à développer une émigration clandestine. Le début des années 1980 vit la fin de la période d'ouverture de la Malaisie à l'émigration indonésienne. Longtemps peu regardantes à l'égard du travail clandestin, les autorités malaysiennes ont, depuis la récession que connut le pays en 1985-1986, considérablement durci leur réglementation en ce domaine. À partir de 1997 et avec la crise économique qui frappa l'Asie du Sud-Est cette même année, le passage à une émigration choisie interdisant, par exemple, aux travailleurs non qualifiés de faire venir leur famille, puis l'adoption de mesures visant à limiter la part des Indonésiens dans la main-d'œuvre immigrée (annonce par le Premier ministre Mahathir de la politique Hire Indonesia Last), ont par ailleurs contribué a augmenter la part de clandestins chez les travailleurs indonésiens ${ }^{21}$.

21 Selon l'Agence nationale pour le placement et la protection des travailleurs indonésiens à l'étranger (BNP2TKI), la Malaisie abritait environ 700000 clandestins en 2008. Une véritable industrie du travail clandestin, souvent dirigée par d'anciens ouvriers indonésiens installés dans la péninsule, s'est mise en place depuis quelques années: recrutés dans l'ensemble des régions de l'Archipel, les candidats à l'émigration passent 
en moyenne par sept intermédiaires avant d'arriver à destination ${ }^{22}$. La traversée du détroit de Malacca, autrefois si facile, est devenue un moment périlleux du voyage. Effectuée de nuit pour éviter les patrouilles, souvent sur des embarcations de fortune, elle est fréquemment l'occasion de drames : entre 1990 et 1995, 5000 Indonésiens se seraient ainsi noyés dans le détroit (Human rigths watch Asia). Ces travailleurs clandestins, privés de droits, sont par ailleurs devenus, ici comme ailleurs, une variable d'ajustement pour les autorités malaysiennes en fonction des conditions économiques et des exigences de l'opinion publique : 10700 Indonésiens ont été renvoyés chez eux en 2005, 35300 en 2008 et, en 2009 ce chiffre était presque dépassé dès le mois de septembre, en raison du ralentissement de l'économie malaisienne ${ }^{23}$.

L'émigration en Malaisie est donc devenue au fil des ans la marque du retard de développement pris par l'Indonésie sur son voisin du nord. Accélérée par la très grave crise qui a frappé le pays entre 1997 et 1999, l'immigration de pauvreté (tant légale qu'illégale) est devenue largement majoritaire. Elle a rapidement posé un certain nombre de problèmes sociaux mais l'Indonésie, alors plongée dans une situation de chaos intérieur, a été incapable de s'en soucier. Depuis quelques années cependant, le retour à une situation plus stable a incité les Indonésiens à reporter leur attention sur le sort réservé à leurs compatriotes expatriés. Le destin de ces travailleurs, privés parfois des droits les plus élémentaires, a suscité un intérêt croissant dans le pays. Le mécontentement indonésien quant à cette situation a trouvé, depuis peu, des prolongements culturels et politiques témoignant d'une frustration croissante à l'égard du voisin malaisien.

\section{La réaffirmation des frontières culturelles et politiques : un exutoire aux frustrations migratoires ?}

Plusieurs affaires sordides de maltraitance de domestiques indonésiennes en Malaisie ont mobilisé l'opinion ces deux dernières années. Les cas semblables signalés dans les pays du Golfe n'ont jamais donné lieu à des campagnes de presse équivalentes à celles qu'a connues la Malaisie ${ }^{24}$ et n'ont pas suscité de réactions officielles.

En juin 2009, à la suite d'un nouveau drame (une domestique torturée pendant trois ans par son employeur malaysien), le gouvernement indonésien, sous la pression de l'opinion publique, a décidé de suspendre l'envoi de tout nouveau travailleur en Malaisie pour exiger la révision d'un accord sur les migrations de travail signé en 2006. Après une année de négociation, la Malaisie a finalement accepté, en mai 2010, de signer une convention garantissant un minimum de droits à ces "héros des devises" (Pahlawan devisa). Ils sont désormais autorisés à conserver leur passeport par devers eux, afin de pouvoir fuir, au besoin, un employeur indélicat ; ils doivent avoir un jour de congé par semaine ; le montant de leur salaire est désormais contrôlé par les deux États qui s'engagent, par ailleurs, à coopérer pour prévenir (et au besoin punir) les abus $^{25}$.

Le sursaut nationaliste anti-malaisien suscité par ces drames n'a pas concerné que cette émigration de pauvreté. Alimentées par un climat de méfiance, les rancœurs indonésiennes se sont également exprimées à propos d'autres affaires et, surtout, la querelle s'est cristallisée depuis quelques mois autour de deux thèmes, celui de l'identité culturelle et celui de la délimitation des frontières. Les changements 
politiques intervenus dans les deux pays, d'abord en Indonésie avec la chute de Suharto en 1998, puis en Malaisie avec le retrait de Mahatir, ont, par ailleurs, privé les relations bilatérales d'une intimité entre des gouvernants se connaissant de longue date et capables de régler leurs différents par des contacts personnalisés.

Depuis plusieurs années, les Indonésiens reprochent ainsi à la Malaisie une série d'emprunts à la culture de l'Archipel, en particulier pour faire la promotion de son patrimoine touristique (carte 4). Les premières plaintes en ce sens dataient de $2005^{26}$, mais les dénonciations se sont multipliées à partir de 2007 et, en 2009, elles furent régulièrement récapitulées à la une des journaux et, surtout, elles furent présentées comme autant de preuves d'un vaste complot visant à priver l'Indonésie de son identité. La Malaisie fut ainsi accusée de vouloir s'approprier des arts traditionnels comme le théâtre d'ombres (wayang kulit) javanais ou la danse pendet de Bali, des chansons («Soleram » de Riau, « Anak Kambing Saya » de Nusa Tenggara), des plats (le rendang) de Sumatra, les poignards sacrés (kriss) de Java ou encore des tissus (kain ulos) de Sumatra Nord. En août 2009, des associations indonésiennes affirmèrent même que l'hymne national malaisien était en fait le plagiat d'un keroncong, une chanson traditionnelle écrite par un ensemble de Bandung, en $1956^{27}$.

Carte 4

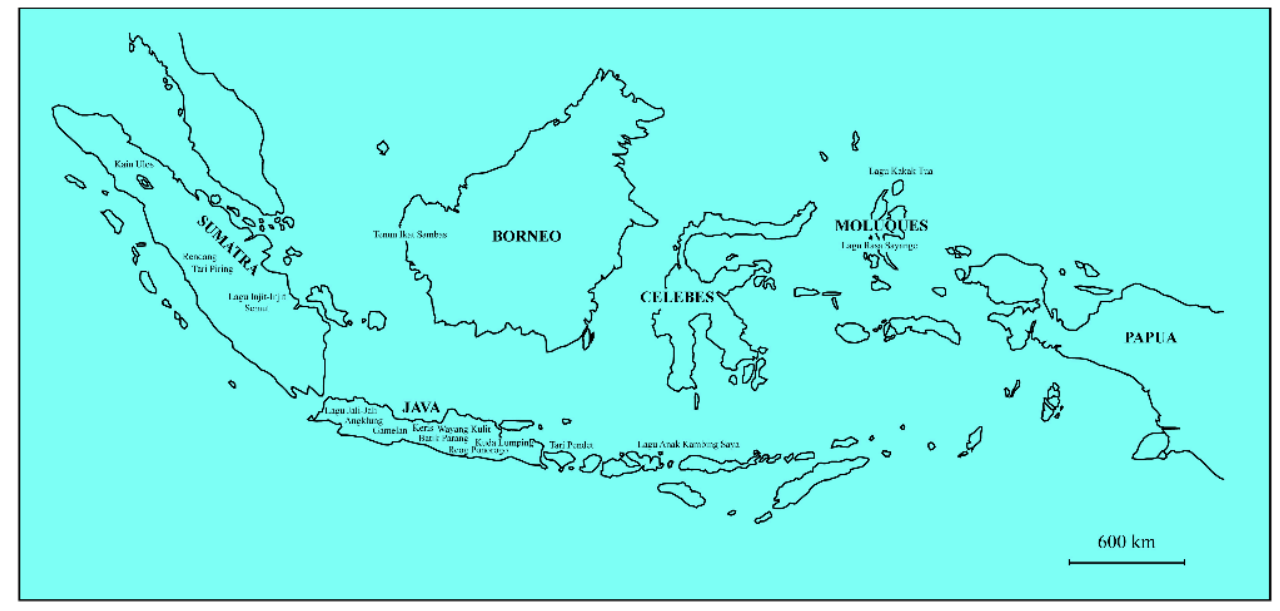

Les « emprunts » culturels malaisiens à l'Indonésie.

Conception Remy Madinier (cNRS-IRASEC), réalisation Ade Pristic Wahyo (EFEO)

27 S'appuyant sur ces divers exemples, une véritable hystérie nationaliste à l'égard de la Malaisie s'est emparée d'une partie de l'opinion publique indonésienne. En septembre 2009, des manifestations inédites ont eut lieu à Jakarta : un groupe ultranationaliste, la Forteresse de la démocratie du peuple (Benteng Demokrasi Rakyat, dont l'acronyme, Bendera, signifie le drapeau), a bloqué, plusieurs heures durant, une grande artère de la capitale afin de rechercher les citoyens malaisiens, dans l'intention de les expulser. Une semaine plus tard, une autre association prétendant, quant à elle, représenter les travailleurs indonésiens émigrés, a défilé, armée de pointes de bambous, au chant de Membonkar Malaysia («détruisons la Malaysie ») ${ }^{28}$. En octobre 2009, fort de son succès médiatique, Bendera a même prétendu préparer une invasion du Sarawak, l'un des États malaisiens de Bornéo ${ }^{29}$. 
Toujours entretenue par la révélation de nouvelles affaires de maltraitance d'émigrés indonésiens, la tension avec la Malaisie a connu, en août 2010, un nouveau rebondissement cette fois lié à l'épineuse question de la délimitation des frontières. Une centaine de kilomètres ( $20 \%$ du total) de frontières maritimes entre les deux pays étant encore l'objet de contentieux, les désaccords sur l'exploitation des «zones grises " sont fréquents ${ }^{30}$. L'arraisonnement par la marine indonésienne de plusieurs bateaux de pêche malaisiens, suivi, quelques jours plus tard, de l'arrestation de plusieurs gardes-frontières indonésiens par la partie adverse, déclenchèrent de virulentes réactions en Indonésie. Des manifestations eurent lieu dans la plupart des grandes villes du pays et, à Jakarta, l'ambassade de Malaisie fut bombardée de matières fécales par les militants du groupe Bendera, sans que la police n'intervienne ni ne poursuive les auteurs de cette provocation ${ }^{31}$. Dans les semaines qui suivirent, l'affaire prit un tour politique et le gouvernement fut accusé, jusque dans la presse modérée, de manquer de fermeté à l'égard de son voisin.

On ne peut comprendre l'ampleur et la virulence de cet accès de fièvre ultranationaliste autour d'un incident de frontière somme toute relativement mineur, en dehors du contexte migratoire évoqué plus haut: les drames liés à cette émigration, régulièrement à la une des journaux du pays, viennent sans cesse rappeler à l'Indonésie son statut d'infériorité économique et sociale à l'égard de la Malaisie. Dans ce contexte tendu, la riposte, tant sur un plan culturel qu'à travers la question des limites territoriales, a permis aux Indonésiens de reprendre à leur compte l'idée de frontières rigides délimitant des espaces à l'identité culturelle clairement identifiée. En d'autres termes, ces querelles ont permis à l'Indonésie d'adopter une posture symbolique dominante face à la fin d'une circulation pluriséculaire des hommes et des idées, au sein d'un espace commun de civilisation. Dans le cas des migrations, au contraire, cette fermeture était imposée par la Malaisie à l'Indonésie et donnait lieu à des échanges fondés sur l'inégalité.

Plus généralement, l'effort de labellisation qu'entreprend désormais le gouvernement indonésien pour l'ensemble des cultures régionales constitue un moyen de s'approprier une modernité dont le brevet et la reconnaissance internationale de la paternité intellectuelle constituent des éléments essentiels. Accusant un retard certain sur le plan technologique par rapport à ses voisins, cantonnée dans un rôle de sous-traitant dans la fabrication de produits conçus ailleurs, l'Indonésie retrouve ici la fierté liée au statut d'inventeur. À ce titre, la reconnaissance par l'UNESCO, en septembre 2009, du batik indonésien comme élément du patrimoine culturel de l'humanité fut présentée comme une victoire sur la Malaisie (qui souhaitait elle aussi voir son artisanat du batik reconnu) et un moyen de rendre, à défaut de justice, leur honneur à tous ces migrants anonymes à l'honneur bafoué32.

\section{Migration et islamisme radical}

31 Il est enfin un autre domaine, celui de l'islamisme radical, où la question de la paternité de phénomènes liés à des circulations migratoires a fait l'objet de récentes querelles. $\mathrm{Au}$ rebours des questions culturelles évoquées plus haut, il s'agit cette fois, pour l'Indonésie, de rejeter sur son voisin malaisien une part de responsabilité dans la formation des réseaux terroristes qui ont mis à mal la sécurité intérieure depuis le 
début des années 2000, ternissant sa réputation internationale et pesant lourdement sur son secteur touristique.

Ainsi, de manière a priori inattendue, un virulent sentiment anti-malaisien en Indonésie s'est exprimé lors de la traque puis de l'élimination, en septembre 2009, de Noordin Mohammad Top, le responsable de l'une des branches de l'organisation terroriste Jemaah Islamiyah, responsable ces dernières années de plusieurs attentats sanglants dans l'Archipel. Le parcours de ce citoyen malaisien illustre en effet le rôle des réseaux migratoires entre l'Indonésie et la Malaisie dans la constitution d'une organisation terroriste régionale. Mais, il constitue aussi pour les Indonésiens le reflet des échanges inégaux entre les deux pays. À partir des années 1980 et jusqu'au 11 septembre 2001, en effet, la Malaisie s'était montrée relativement accueillante à l'égard des organisations islamistes radicales du monde entier. Elle avait en particulier hébergé sur son sol Abdullah Sungkar et Abu Bakar Ba'asyir, deux Indonésiens pourchassés dans leur pays qui furent à l'origine de la création de la Jemaah Islamiyah. Rassemblant d'anciens jihadistes formés en Afghanistan, cette organisation entendait porter la guerre sainte dans plusieurs pays de la région, le sud des Philippines et l'Indonésie en particulier ${ }^{33}$. Alors qu'aucun attentat n'a jamais eu lieu en Malaisie, l'Indonésie a, quant à elle, été durement touchée. Pour bien des Indonésiens, la responsabilité de leur voisin dans la vague de violence religieuse qui a ébranlé le pays est le reflet d'une situation dans laquelle la Malaisie s'approprie ce qui l'arrange mais exporte ses problèmes. À l'instar des affaires de maltraitance envers les employés de maison et de renvoi des travailleurs illégaux, la concomitance entre les reproches liés aux radicaux musulmans et la querelle culturelle fut tout à fait significative. Le slogan popularisé par les cybernautes indonésiens après les attentats de juillet 2009 à Jakarta, "Noordin M Top est à vous, le Pendet est à nous ", témoignait des liens établis dans l'opinion publique entre ces deux affaires. Comme dans le cas d'une main-d'œuvre exploitée puis rejetée, le fait que les Malaisiens aient pu utiliser pour la promotion de leur tourisme une danse originaire de l'île de Bali (le Pendet) frappée, en 2002 et 2005, par deux attentats meurtriers, est désormais perçu comme le signe d'un impérialisme réclamant un sursaut de l'identité nationale et un contrôle des frontières symboliques trop longtemps poreuses.

$\mathrm{Au}$ total, si la question des migrations entre Indonésie et Malaisie semble bien constituer la matrice des mouvements anti-malaisiens de ces dernières années, c'est parce qu'elle a entraîné et diffusé, jusque dans les régions reculées de l'Archipel, une prise de conscience tardive des inégalités de développement entre les deux pays. Cette rupture dans l'histoire des processus migratoires, autrefois souples et désormais déclinés sur un mode plus douloureux, a nourri un complexe d'infériorité, favorisé par les tentatives d'appropriation par la Malaisie d'une identité culturelle autrefois librement partagée. 


\section{NOTES}

1. Pour une réflexion approfondie sur la question de l'identité malaise voir Nathalie Fau, «Être malais de part et d'autre du détroit de Malacca : approche transfrontalière ", Aséanie, 23, juin 2009 : 107-144. Je tiens ici à remercier Nathalie Fau pour ses suggestions éclairées et sa lecture critique d'une précédente version de cette contribution.

2. Sur ces royaumes voir Nicholas Tarling (ed.), The Cambridge History of Southeast Asia, Cambridge, Cambridge University Press, 1999, volume 1, From Early Times to c.1500, et en particulier le chapitre 3 «The Early Kingdoms » (Keith W. Taylor).

3. Denys Lombard, Le Carrefour javanais. Essai d'histoire globale, tome 2, Les réseaux asiatiques, Paris, Éditions de l'EHESS, 1990.

4. Muriel Charras et Manuelle Franck, "Quarante ans d'introversion en Indonésie, l'éclipse de toute une région: l'Asie du Sud-Est insulaire dans les recompositions spatiales asiatiques », in Françoise Cayrac Blanchard, Stéphane Dovert et Frédéric Durand (éd.), Indonésie, un demi-siècle de construction nationale, Paris, L'Harmattan, $2000:$ 69-107.

5. Sur cette possibilité d'appropriation de l'identité malaise, en particulier à travers l'islamisation, voir par exemple le cas de Sumatra Nord, étudié par Daniel Perret: La Formation d'un paysage ethnique. Batak et Malais de Sumatra Nord-Est, Paris, Presses de l'École française d'Extrême-Orient, 1995, 374 p.

6. Nathalie Fau, «D'une rive à l'autre : les migrations entre l'Indonésie et la Malaisie dans le détroit de Malacca », Revue européenne des migrations internationales,(16) 1, 2000 : 151-181.

7. Ibid.

8. Amarjit Kaur, «Mobility, labour mobilisation and border controls: Indonesian labour migration to Malaysia since 1900 ",contribution pour la $15^{\text {th }}$ Biennial Conference of the Asian Studies Association of Australia in Canberra 29 June-2 July 2004.

9. Nathalie Fau, «Être malais de part et d'autre du détroit de Malacca: approche transfrontalière ", op. cit.

10. Amarjit Kaur, «Mobility, labour mobilisation and border controls: Indonesian labour migration to Malaysia since 1900 ", op. cit. À la même époque cependant, une nouvelle conception du monde malais apparaît: d'inspiration nationaliste, elle vise à le réduire à la seule péninsule. Nathalie Fau, « Être malais de part et d'autre du détroit de Malacca : approche transfrontalière », op. cit.

11. Les 1,2 million d'habitants du Sabah et du Sarawak reçurent 40 sièges au Parlement fédéral alors que les 1,7 million de Singapouriens n'étaient représentés que par 15 parlementaires.

12. Sur ce sujet voir Romain Bertrand, État colonial, noblesse et nationalisme à Java. La tradition parfaite, Khartala, Paris, Karthala (Recherches internationales), 2005.

13. Nathalie Fau, «D'une rive à l'autre: les migrations entre l'Indonésie et la Malaisie dans le détroit de Malacca », op. cit.

14. The Cambridge History of Southeast Asia, op. cit., vol 2, chapitre $3: 139$ et suivantes.

15. Muriel Charras et Manuelle Franck, «Quarante ans d'introspection en Indonésie : l'éclipse de toute une région, l'Asie du Sud-Est insulaire dans les recompositions spatiales asiatiques ", op. cit. ; Graeme Hugo, "Indonesian labour migration to Malaysia : trends and policy implications ", Southeast Asian Journal of Social Science,21, $\mathrm{n}^{\circ}$ 1, 1993 : 36-70. Relativement stable, le différentiel de coût de la main-d'œuvre entre l'Indonésie et Singapour représente, depuis une vingtaine d'année, un facteur de 1 à 4 , la Malaysie se situant dans une position intermédiaire.

16. Amarjit Kaur, op. cit.

17. World Bank, The Malaysia-Indonesia Remittance Corridor, Working Paper $n^{\circ}$ 149, 2008. 
18. Chiffre de 2006, Department of Statistics Malaysia, "International migrations in Malaysia », 2007.

19. Azizah Kassim, "The unwelcome guest: Indonesian immigrants and Malaysian public responses », Southeast Asian Studies, 25, n 2, 1987 : 265-278.

20. En 2002 environ $36 \%$ de ces indonésiens employés en Malaisie travaillaient dans le secteur secondaire, $26 \%$ dans l'agriculture, $23 \%$ étaient des travailleurs domestiques et $8 \%$ étaient dans le bâtiment (kaur 2004).

21. Joseph Liow, « Malaysia's illegal Indonesian migrant labour problem : in search of solutions ", Contemporary Southeast Asia, vol. 25, 2003.

22. Nathalie Fau, op. cit.

23. Jakarta Golbe, 20 novembre 2009.

24. Selon la presse près de $60 \%$ des employés de maison expatriés dans le monde rencontreraient des problèmes de mauvais traitement ou de non-paiement de leurs gages et les problèmes de maltraitance dans les pays du Golfe, bien que peu relayés dans la presse, restent très nombreux. Jakarta Globe, 26 août 2009.

25. Kompas 19 mai 2010, « Perlindungan TKI diperkuat », (La protection des travailleurs émigrés renforcés).

26. Kompas, 31 août 2009.

27. Il apparut par la suite que l'œuvre originale qui avait inspiré les deux versions incriminées était une chanson du musicien français Pierre-Jean de Béranger, composée à la fin du XIX ${ }^{\mathrm{e}}$ siècle.

28. Kompas, 14 septembre 2009.

29. Jakarta Globe, 13 octobre 2009.

30. Kompas, 6 septembre 2010

31. Soutenu par plusieurs associations, dont le Forum Betawi Rempug (Forum d'amitié jakartanais), le porte-parole de Bendera justifia cette action par le fait que la Malaisie « abusait de nos travailleurs émigrés et piétinaient notre intégrité », Jakarta Globe, 30 août 2010. Dans les semaines qui suivirent des slogans hostiles à la Malaisie fleurirent sur les murs de Jakarta dont certains («Ganyang Malaysia », « À bas la Malaisie ») faisaient clairement référence à la période de la Konfrontasi.

32. Tempo, 20 septembre 2009.

33. Stéphane Dovert et Rémy Madinier, Les musulmans d'Asie du Sud-Est face aux vertiges de la radicalisation, ouvrage coordonné et rédigé en collaboration, IRASEC-Les Indes savantes, 2003; Rémy Madinier, «Flux et réseaux du radicalisme musulman en Asie du Sud-Est, une approche historique », in Bernard Jouve et Yann Roche (éd.), Géographie politique des flux globaux, Sainte-Foy, Presses de l'université du Québec, 2006 : 251-271.

\section{RÉSUMÉS}

Malgré une longue histoire commune et bien qu'appartenant tous deux à la même aire culturelle (le monde malais) l'Indonésie et la Malaisie, du fait de leurs passés coloniaux respectifs et de leurs évolutions économiques et démographiques depuis les indépendances, ont connu des modes de développement très différents. Très largement fondée, depuis la colonisation anglaise, sur une importation massive de main-d'oeuvre étrangère, la remarquable insertion de la Malaisie dans l'économie mondiale a suscité, depuis plusieurs décennies, une immigration indonésienne 
massive. L'immense majorité du million de travailleurs présents (légalement ou illégalement) en Malaisie est aujourd'hui originaire d'Indonésie. De nombreux travaux universitaires récents (tant locaux qu'occidentaux) permettent de mieux comprendre les aspects démographiques et économiques de ces phénomènes migratoires. Tout en rappelant les principaux résultats de ces travaux, cette communication entend s'intéresser, à partir de l'étude de sources locales, tant indonésiennes que malaisiennes, aux enjeux culturels, religieux et politiques de ces migrations. La présence massive d'Indonésiens au-delà du détroit de Malacca a été en effet longtemps perçue par les Malaisiens comme une alternative satisfaisante aux immigrations indienne et chinoise de la période coloniale. La population malaise ( $60 \%$ des 27 millions de Malaisie) et les autorités ont longtemps considéré que, pour des raisons de proximité religieuse, culturelle et linguistique, l'assimilation des populations issues de son imposant voisin (240 millions d'habitants) se ferait plus aisément. Ces dernières années cependant, des questions religieuses (leadership du "nouvel islam asiatique", réseaux radicaux,...), culturelles (revendication par la Malaisie à des fins touristiques de traditions de certaines régions d'Indonésie) et sociaux (sort des employées de maison indonésiennes en Malaisie, renvois massifs en Indonésie d'immigrants illégaux en période de crise) sont venus singulièrement compliquer la gestion de ces migrations par les deux États. Les enjeux économiques qui jusque-là prévalaient dans l'organisation de ces migrations ont été relégués au second plan et les autorités doivent désormais compter avec de violentes campagnes de presse qui menacent jusqu'à leurs relations diplomatiques.

Indonesia and Malaysia have a long shared history and belong to the same culture area (the Malay world). However, their respective colonial pasts and economic and demographical evolutions since gaining independence mean that both countries have developed very differently. Since Malaysia was colonised by the English, it has relied on the massive importation of foreign labour to take its place convincingly in the global economy. In the last few decades, this has led to a flood of Indonesian immigrants. Today, the vast majority of the one million workers living in Malaysia (legally or illegally) are of Indonesian origin. Many recent academic studies (by local and Western researchers) focus on the demographic and economic reasons behind these migratory movements. This article presents the main conclusions from this body of research and, using local Indonesian and Malaysian sources, identifies the cultural, religious and political issues caused by migration. The wave of Indonesians crossing the Strait of Malacca was long considered by Malaysians as a satisfactory alternative to Indian and Chinese immigration during the colonial era. For a long time, the Malay people (60\% of 27 million Malaysians) and Malay authorities thought that the assimilation of immigrants from Indonesia (which has a total population of 240 million) would be easier for religious, cultural and linguistic reasons. However, in recent years, managing these migratory flows has become more complicated for both countries, because of issues to do with religion (the leadership of the new Asian Islam, radical networks, etc.), culture (Malaysia has used traditions and customs from some Indonesian regions for tourism purposes) and society (the fate of Indonesian homeworkers in Malaysia, the mass deportation of illegal Indonesian immigrants during the economic crisis, etc.). The economic issues that previously dominated migratory management have become secondary. Authorities must now deal with virulent press campaigns that go so far as to threaten diplomatic relations.

\section{INDEX}

Thèmes : migration, mobilité

Index géographique : Indonésie, Malaisie 


\section{AUTEUR}

\section{RÉMY MADINIER}

Historien et spécialiste de l'Islam du monde malais, chargé de recherche au CNRS-USR 3142, Institut de recherche sur l'Asie du Sud-Est contemporaine (IRASEC-UMIFRE 22), Bangkok, http://www.irasec.com/, en résidence à Jakarta depuis août 2008.

remy.madinier@gmail.com 